\title{
Slant Helices that Constructed from Hyperspherical Curves in the n-dimensional Euclidean Space
}

\author{
Bülent Altunkaya \\ (Communicated by Kazım İlarslan)
}

\begin{abstract}
In this work, we study slant helices in the n-dimensional Euclidean space. We give methods to determine the position vectors of slant helices from arclength parameterized curves that lie on the unit hypersphere. By means of these methods, first we characterize slant helices and Salkowski curves which lie on 2 -dimensional hyperboloid. After that, we characterize rectifying slant helices which are geodesics of 2 -dimensional cone.
\end{abstract}

Keywords: Slant helix, Salkowski curve, rectifying curve, hyperspherical curve, geodesic of a hypersurface.

AMS Subject Classification (2010): 53A04, 53C40.

\section{Introduction}

The notion of slant helix in the Euclidean 3-space has been introduced by Izumiya and Takeuchi in [14]. Slant helices defined by the property that normal lines of a regular curve make a constant angle with a fixed direction. They also gave some characterizations of these curves. After that, slant helices in the Euclidean 3-space were studied by other researchers from different fields [3, 15, 16, 17].

The notion of slant helix in Euclidean 3-space can be generalized to higher dimensions by using the same definition [1,22]. In [1], authors gave some necessary and sufficient conditions for a curve to be a slant helix in Euclidean n-space.

If the position vector of a space curve always lies in its rectifying plane, we call it a rectifying curve. Such curves are first studied by Chen [7, 8]. Later, he stated that a curve on a cone in Euclidean 3-space is a geodesic if and only if it is either a rectifying curve or an open portion of a ruling in [10]. Because of the importance of these curves, a rich literature has been created by different researchers [9, 10, 11, 13, 23]. Afterwards, authors extended the rectifying curve concept to upper dimensions $[6,18]$.

About a century ago, E. Salkowski introduced a family of space curves (that would later be called Salkowski curves [21]) with constant curvature and non-constant torsion. Recently, J. Monterde showed that these curves are also slant helices [19]. Although, there are many studies about Salkowski curves in the 3-dimensional Euclidean space, there are no characterizations or examples about these curves in n-dimensional Euclidean space when $n>3$.

Despite the studies on these subjects, the position vectors of slant helices, rectifying slant helices and Salkowski curves couldn't be generalized the n-dimensional Euclidean space. In this work, we give methods to determine the position vectors of such curves by using arclength parameterized curves that lie on the unit hypersphere for $n \geq 3$. In addition, we introduce families of rectifying slant helices that lie on cones which are also geodesics of these cones. We also introduce slant helix families that lie on hyperboloids. 


\section{Preliminaries}

Let $\mathbb{E}^{n}$ denote the n-dimensional Euclidean space with the standart orthonormal basis $\left\{e_{1}, e_{2}, \ldots, e_{n}\right\}$, that is, $\mathbb{R}^{n}$ equipped with the standard inner product

$$
\langle x, y\rangle=\sum_{i=1}^{n} x_{i} y_{i}
$$

for each $x=\left(x_{1}, x_{2}, \ldots, x_{n}\right), y=\left(y_{1}, y_{2}, \ldots, y_{n}\right) \in \mathbb{R}^{n}$ where the norm of a vector $x \in \mathbb{R}^{n}$ is given by $\|x\|^{2}=$ $\langle x, x\rangle$.

Similar to space curves; for a regular curve $\alpha: I \subset R \rightarrow \mathbb{E}^{n}$, we have the Frenet frame $V_{1}, V_{2}, \ldots, V_{n}$ that satisfy the equations

$$
\left\{\begin{array}{c}
V_{1}^{\prime}(t)=\nu(t) k_{1}(t) V_{2}(t) \\
V_{i}^{\prime}(t)=\nu(t)\left(-k_{i-1}(t) V_{i-1}(t)+k_{i}(t) V_{i+1}(t)\right), \quad i=2,3, \ldots, n-1 \\
V_{n}^{\prime}(t)=-\nu(t) k_{n-1}(t) V_{n-1}(t)
\end{array}\right.
$$

where $\nu(t)=\|d \beta(t) / d t\|=\left\|\beta^{\prime}(t)\right\|$ and $k_{i}, i \in\{1,2, \ldots, n-1\}$ denote the $i$ th curvature function of the curve $\beta$ [12].

From Equation 2.1; if $k_{n-1}(t)=0$ for all $t \in I$, then the curve $\beta$ lies in the orthogonal complement of $V_{n}$. Therefore, we can say $\beta$ lies in a $(n-1)$-dimensional hyperplane which is perpendicular to $V_{n}$.

Similar to above, if the position vector of a curve $\beta$ lies in the orthogonal complement of $V_{2}$ which is

$$
V_{2}^{\perp}(t)=\left\{v \in T_{\beta(t)} \mathbb{E}^{n} \mid \quad\left\langle v, \quad V_{2}(t)\right\rangle=0\right\},
$$

then we call it a rectifying curve [6]. Therefore, we state rectifying curve definition as follows.

Definition 2.1. A curve $\beta: I \rightarrow \mathbb{E}^{n}$ is called rectifying curve if, for all $t \in I$, the orthogonal complement of $V_{2}$ contains a fixed point [5].

Note that this definition is independent from the coordinates. A necessary and sufficient condition for a unit speed curve to be a rectifying curve is that

$$
\left\langle\beta(s), V_{1}(s)\right\rangle=s+c
$$

for some constant $c \in R$ where $s$ is the arclength parameter of the curve $\beta$ [5]. We extend the condition in Equation 2.2 for arbitrary speed curves in Corollary 3.2.

In $\mathbb{E}^{3}$, a curve $\alpha$ with $k_{1}(t) \neq 0$ is called a slant helix if the principal normal lines of the curve $\beta$ make a constant angle with a fixed direction [14]. Therefore, we will use the definition below to show the curve $\beta$ is a slant helix.

Definition 2.2. A curve $\beta: I \rightarrow \mathbb{E}^{n}$ is called slant helix if its principal normal $V_{2}$ makes a constant angle with a fixed direction [1].

Salkowski curves, a space curve family with constant curvature and non-constant torsion, are defined in [21]. From the paper [19], we know this family of curves are also slant helices. If we combine these two concepts, we can give the Salkowski curve definition in $\mathbb{E}^{n}$ as follows.

Definition 2.3. A slant helix $\beta: I \rightarrow \mathbb{E}^{n}$ is called a Salkowski curve if the first curvature function is a constant function and the other curvature functions are non-constant functions.

In this study, we work with non-degenerate curves which means $k_{i}(t) \neq 0$ for all $t \in I$ and $i \in$ $\{1,2, \ldots, n-1\}$.

\section{Methods of constructing slant helices in $\mathbb{E}^{n}$}

In this section, we give methods to develope different type of slant helices. We characterize slant helices by constructing them starting from an arclength parameterized curve on the unit hypersphere. Our first characterization is as follows. 
Theorem 3.1. Let $\gamma(u): I \rightarrow \mathbb{E}^{n}$ be a unit speed spherical curve on $S^{n-1}$ with

$$
\left\|\frac{d^{2} \gamma(u)}{d u^{2}}\right\|=a
$$

such that

$$
\gamma(u)=\left(\gamma_{1}(u), \gamma_{2}(u), \ldots, \gamma_{n-1}(u), c\right)
$$

where $u(t): I \rightarrow R$ is a differentiable function, $a>1$ and $c \in R /\{0\}$. Then, the curve $\beta$ defined by

$$
\beta(t)=\int \alpha(u(t)) d t
$$

is a unit speed slant helix in $\mathbb{E}^{n}$ where

$$
\alpha(u)=\sin (u) \gamma(u)+\cos (u) \frac{d \gamma(u)}{d u}
$$

Proof. From the definitions of the curve $\gamma$, we have

$$
\|\gamma(u)\|=1, \quad\left\|\frac{d \gamma(u)}{d u}\right\|=1, \quad\left\|\frac{d^{2} \gamma(u)}{d u^{2}}\right\|=a .
$$

With straightforward computations, we have the spherical general helix $\alpha$ (See [4]) with

$$
\|\alpha(u)\|=1, \quad\left\|\frac{d \alpha(u)}{d u}\right\|=\sqrt{a-1} \cos (u) .
$$

Therefore, $\beta$ is a unit speed curve with the first curvature function

$$
k_{1}^{\beta}=\sqrt{a-1} \cos (u) u^{\prime}
$$

where $\sqrt{a-1}=\left\|\frac{d^{2} \gamma(u)}{d u^{2}}+\gamma(u)\right\|$.

If we compute the first two Frenet vectors of the curve $\beta$, we have

$$
\begin{gathered}
V_{1}^{\beta}=\alpha(u), \\
V_{2}^{\beta}=\frac{1}{\sqrt{a-1}}\left(\gamma(u)+\frac{d^{2} \gamma(u)}{d u^{2}}\right) \\
=\frac{1}{\sqrt{a-1}}\left(\gamma_{1}(u)+\frac{d^{2} \gamma_{1}(u)}{d u^{2}}, \ldots, \gamma_{n-1}(u)+\frac{d^{2} \gamma_{n-1}(u)}{d u^{2}}, c\right) .
\end{gathered}
$$

Therefore, $\beta$ is a unit speed slant helix whose second Frenet vector makes the constant angle $\theta=$ $\arccos \left(\frac{c}{\sqrt{a-1}}\right)$ with $e_{n}$.

Now, we give an important result of the Theorem 3.1. In order to reach our goal, we use W-curves, i.e. a curve which has constant Frenet curvatures [2,5].

Lemma 3.1. Consider the $W$-curve $\gamma(u): I \rightarrow S^{2 n-1}(P, R) \subset S^{2 n} \subset \mathbb{E}^{2 n+1}$ such that

$$
\gamma(u)=\frac{R}{\sqrt{n}}\left(\sum_{j=1}^{n} \cos \left(c_{j} u\right) e_{2 j-1}+\sum_{j=1}^{n} \sin \left(c_{j} u\right) e_{2 j}\right)+\sqrt{1-R^{2}} e_{2 n+1}
$$

where $u: I \rightarrow R$ is a differentiable function, $P=\left(0,0, \ldots, 0, \sqrt{1-R^{2}}\right) \in \mathbb{E}^{2 n+1}, 0<R=\left(\frac{n}{\sum_{j=1}^{n} c_{j}^{2}}\right)^{1 / 2}<1$ and $c_{i}, c_{j} \in R /\{-1,0,1\}, c_{i} \neq c_{j}, 1 \leq i<j \leq n$. Then, the curve $\beta$ defined by

$$
\beta(t)=\int \alpha(u(t)) d t
$$


is a slant helix in $\mathbb{E}^{2 n+1}$ where

$$
\alpha(u)=\sin (u) \gamma(u)+\cos (u) \frac{d \gamma(u)}{d u}
$$

is a spherical general helix.

Proof. With straightforward calculations, we have

$$
\|\gamma(u)\|=1,\left\|\frac{d \gamma(u)}{d u}\right\|=1,\left\|\frac{d^{2} \gamma(u)}{d u^{2}}\right\|=\left(\frac{\sum_{i=1}^{n} c_{i}{ }^{4}}{\sum_{i=1}^{n} c_{i}{ }^{2}}\right)^{1 / 2} .
$$

Hence; $\gamma$ satisfies the conditions in Theorem 3.1. Therefore, $\beta$ is a unit speed slant helix in $\mathbb{E}^{2 n+1}$.

The Corollary below is a compile of interesting results of Lemma 3.1.

Corollary 3.1. If we take $u(t)=t$ in Lemma 3.1, then we have the following results;

(i) The slant helix $\beta$ lies on the hyperboloid

$$
\begin{aligned}
H^{2 n}= & \left\{\left(x_{1}, x_{2}, \ldots, x_{2 n+1}\right) \in \mathbb{E}^{2 n+1} \mid\right. \\
& \left.\frac{\left(\sum_{i=1}^{n} c_{i}^{2}\right)}{4 n}\left[\sum_{j=1}^{n}\left(\frac{c_{j}^{2}-1}{c_{j}^{2}}\right)^{2}\left(x_{2 j-1}^{2}+x_{2 j}^{2}\right)-\frac{\sum_{i=1}^{n}\left(\frac{c_{j}^{2}-1}{c_{j}^{2}}\right)^{2}}{-n+\sum_{i=1}^{n} c_{i}^{2}} x_{2 n+1}\right]=1\right\} .
\end{aligned}
$$

(ii) For $n \geq 2$, if $c_{j}=c_{k}, 1 \leq j, k \leq n, j \neq k$. The slant helix $\beta$ is included in the (2n-1)-dimensional hyperplane of $\mathbb{E}^{2 n+1}$, so we can consider it to be a slant helix in $\mathbb{E}^{2 n-1}$. Also, the slant helix $\beta$ lies on the hyperboloid

$$
H^{2 n-2}=\left\{\left(x_{1}, x_{2}, \ldots, x_{2 n+1}\right) \in H^{2 n} \mid x_{2 j-1}=x_{2 k-1}, x_{2 j}=x_{2 k}\right\} .
$$

(iii) For $n \geq 2$ and $j \in\{1,2, \cdots, n\}$. If $\left|c_{j}\right|=1$, then $(2 j-1)$ th term of the curve $\beta$ vanishes and the (2j)th term of the curve $\beta$ becomes a linear function of the parameter $t$. Therefore, it is included in the $2 n$-dimensional hyperplane of $\mathbb{E}^{2 n+1}$ and its first Frenet vector makes a constant angle with $e_{2 j-1}$. So, we can consider it to be a general helix in $\mathbb{E}^{2 n}$.

Example 3.1. Let us take

$$
n=2, c_{1}=1, c_{2}=4, u(t)=t
$$

in Lemma 3.1. Then, we have the $\mathrm{W}$-curve

$$
\gamma(u(t))=\left(\frac{\cos (t)}{\sqrt{5}}, \frac{\sin (t)}{\sqrt{5}}, \frac{\cos (2 t)}{\sqrt{5}}, \frac{\sin (2 t)}{\sqrt{5}}, \sqrt{\frac{3}{5}}\right)
$$

With straightforward calculations, we find

$$
\beta(t)=\left(0, \frac{t}{\sqrt{17}}, \frac{25 \cos (3 t)+9 \cos (5 t)}{30 \sqrt{17}}, \frac{25 \sin (3 t)+9 \sin (5 t)}{30 \sqrt{17}},-\sqrt{\frac{15}{17}} \cos (t)\right) .
$$

We can consider the curve $\beta$ as

$$
\beta(t)=\left(\frac{t}{\sqrt{17}}, \frac{25 \cos (3 t)+9 \cos (5 t)}{30 \sqrt{17}}, \frac{25 \sin (3 t)+9 \sin (5 t)}{30 \sqrt{17}},-\sqrt{\frac{15}{17}} \cos (t)\right) .
$$

If we compute the Frenet vectors and curvature functions of the curve $\beta$, we have

$$
V_{1}^{\beta}(t)=\left(\frac{1}{\sqrt{17}},-\frac{5 \sin (3 t)+3 \sin (5 t)}{2 \sqrt{17}}, \frac{4 \cos ^{3}(t)(3 \cos (2 t)-2)}{\sqrt{17}}, \sqrt{\frac{15}{17}} \sin (t)\right),
$$




$$
\begin{gathered}
V_{2}^{\beta}(t)=\left(0,-\frac{\sqrt{15}}{4} \cos (4 t),-\frac{\sqrt{15}}{4} \sin (4 t), \frac{1}{4}\right), \\
V_{3}^{\beta}(t)=\left(\frac{4 \cos (t)}{\sqrt{17} \sqrt{9-8 \cos (2 t)}}, \frac{-5 \sin (2 t)+9 \sin (4 t)-3 \sin (6 t)}{\sqrt{17} \sqrt{9-8 \cos (2 t)}},\right. \\
\left.\frac{5 \cos (2 t)-9 \cos (4 t)+3 \cos (6 t)}{\sqrt{17} \sqrt{9-8 \cos (2 t)}}, \frac{2 \sqrt{\frac{15}{17}} \sin (2 t)}{\sqrt{9-8 \cos (2 t)}}\right) . \\
V_{4}^{\beta}(t)=\frac{1}{\sqrt{9-8 \cos (2 t)}}\left(-4 \sin (t), \frac{1}{4} \cos (4 t), \frac{1}{4} \sin (4 t), \frac{\sqrt{15}}{4}\right), \\
k_{1}^{\beta}(t)=4 \sqrt{\frac{15}{17}} \cos (t), \\
k_{2}^{\beta}(t)=\sqrt{\frac{15}{17}} \sqrt{9-8 \cos (2 t)}, \\
k_{3}^{\beta}(t)=\sqrt{\frac{15}{17}} \sqrt{9-8 \cos (2 t)} .
\end{gathered}
$$

Therefore, $\beta$ is a general helix whose first Frenet vector makes the constant angle $\theta=\arccos \left(\frac{1}{\sqrt{17}}\right)$ with $e_{1}$.

Example 3.2. Given that

$$
n=1, c_{1}=2, u(t)=t
$$

in Lemma 3.1. Then, we have the $\mathrm{W}$-curve

$$
\gamma(u(t))=\left(\frac{1}{2} \cos (2 t), \frac{1}{2} \sin (2 t), \frac{\sqrt{3}}{2}\right)
$$

Therefore, we have the spherical general helix (See Figure 1)

$$
\alpha(u(t))=\left(\frac{1}{4}(-3 \sin (t)-\sin (3 t)), \cos ^{3}(t), \frac{\sqrt{3}}{2} \sin (t)\right) .
$$

Consequently, we have the slant helix (See Figure 2)

$$
\beta(t)=\frac{1}{12}((9 \cos (t)+\cos (3 t)),(9 \sin (t)+\sin (3 t)),-6 \sqrt{3} \cos (t))
$$

that lies on the hyperboloid of one sheet

with the Frenet vectors

$$
\frac{9}{4}\left(x_{1}^{2}+x_{2}^{2}\right)-\frac{3 x_{3}^{2}}{4}=1
$$

$$
\begin{gathered}
V_{1}^{\beta}(t)=\left(-\frac{1}{4}(3 \sin (t)+\sin (3 t)), \cos ^{3}(t), \frac{1}{2} \sqrt{3} \sin (t)\right), \\
V_{2}^{\beta}(t)=\left(-\frac{\sqrt{3}}{2} \cos (2 t),-\sqrt{3} \sin (t) \cos (t), \frac{1}{2}\right), \\
V_{3}^{\beta}(t)=\left(-\frac{1}{2} \cos (t)(\cos (2 t)-2), \sin ^{3}(t), \frac{\sqrt{3}}{2} \cos (t)\right)
\end{gathered}
$$

and the curvature functions

$$
\begin{aligned}
& k_{1}^{\beta}(t)=\sqrt{3} \cos (t), \\
& k_{2}^{\beta}(t)=\sqrt{3} \sin (t) .
\end{aligned}
$$




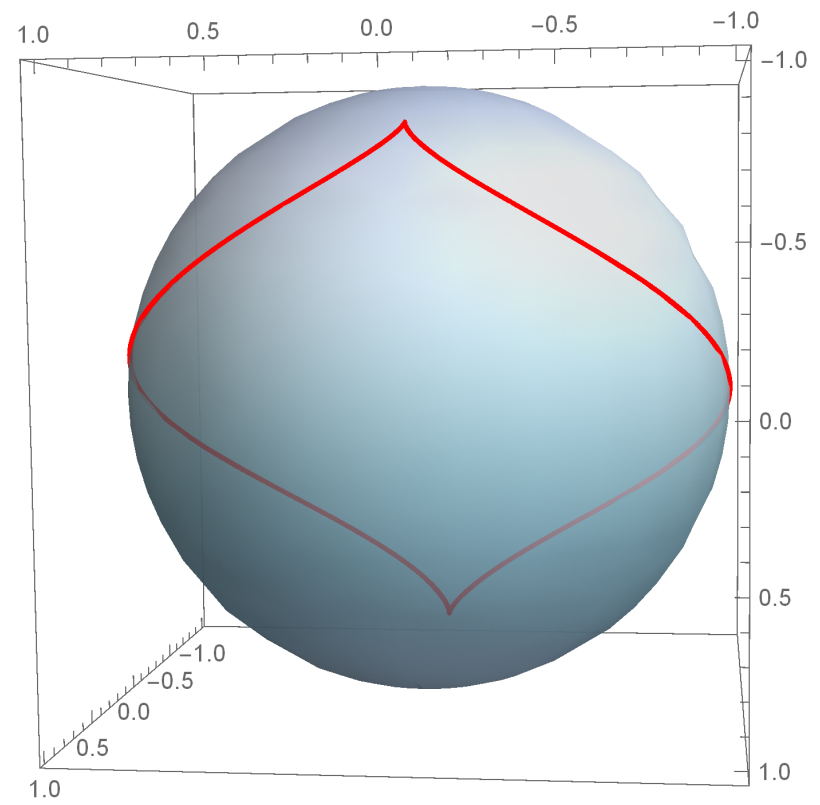

Figure 1. The spherical helix $\alpha$ on $S^{2}$.

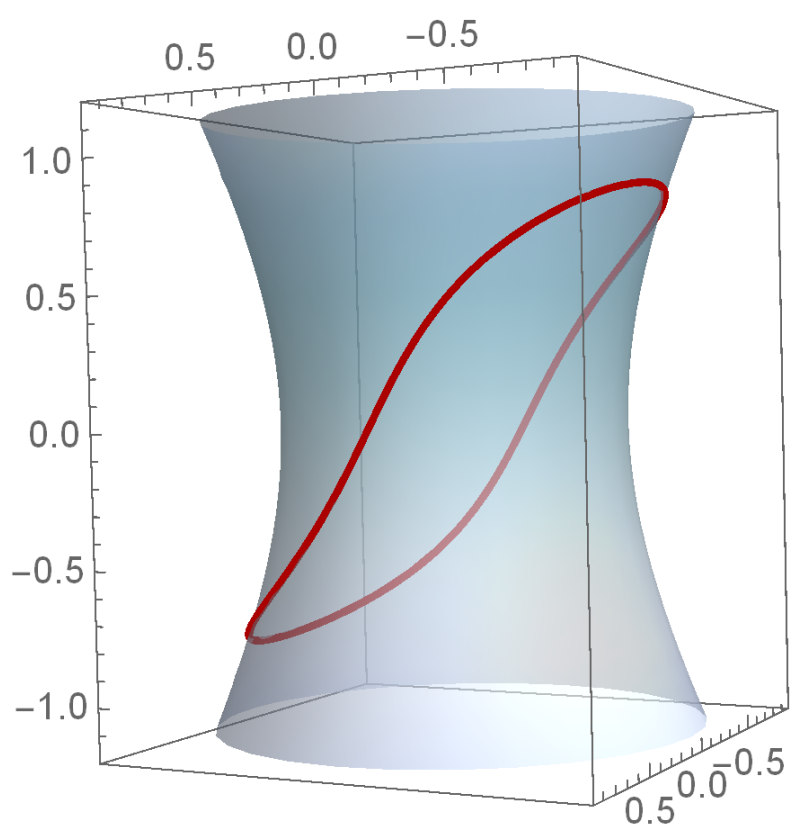

Figure 2. The slant helix $\beta$ on the one sheeted hyperboloid $\frac{9}{4}\left(x_{1}^{2}+x_{2}^{2}\right)-\frac{3 x_{3}^{2}}{4}=1$.

In Definition 2.3, we defined Salkowski curves. From the method introduced in Theorem 3.1, we can construct Salkowski curves in $\mathbb{E}^{n}$ by the Lemma below.

Lemma 3.2. Let $\beta$ be a curve defined by Theorem 3.1. $\beta$ is a Salkowski curve if and only if $u(t)= \pm \arcsin \left(\frac{t}{\sqrt{a-1}}+a_{1}\right)$ where $a_{1} \in R$.

Proof. From Equation 3.1, we have

$$
k_{1}^{\beta}=\sqrt{a-1} \cos (u) u^{\prime}
$$

By solving the differential equation

$$
\sqrt{a-1} \cos (u) u^{\prime}=1
$$


we have

$$
u(t)= \pm \arcsin \left(\frac{t}{\sqrt{a-1}}+a_{1}\right)
$$

Example 3.3. Given that $n=2, c_{1}=3, c_{2}=4$ in Lemma 3.1. Then, we have the $\mathrm{W}$-curve

$$
\gamma(u(t))=\left(\frac{1}{5} \cos (3 t), \frac{1}{5} \sin (3 t), \frac{1}{5} \cos (4 t), \frac{1}{5} \sin (4 t), \frac{\sqrt{23}}{5}\right) .
$$

Consequently, we have the spherical general helix

$$
\begin{aligned}
\alpha(u(t))=( & \frac{1}{5} \sin (u(t)) \cos (3 u(t))-\frac{3}{5} \sin (3 u(t)) \cos (u(t)), \\
& \frac{1}{5} \sin (u(t)) \sin (3 u(t))+\frac{3}{5} \cos (u(t)) \cos (3 u(t)), \\
& \frac{1}{5} \sin (u(t)) \cos (4 u(t))-\frac{4}{5} \sin (4 u(t)) \cos (u(t)), \\
& \frac{1}{5} \sin (u(t)) \sin (4 u(t))+\frac{4}{5} \cos (u(t)) \cos (4 u(t)), \\
& \left.\frac{1}{5} \sqrt{23} \sin (u(t))\right) .
\end{aligned}
$$

By means of Lemma 3.2, if we take $a_{1}=0$, then

$$
u(t)=\arcsin \left(\frac{5 t}{2 \sqrt{78}}\right) .
$$

Therefore, we have the unit speed Salkowski curve $\beta$ in $\mathbb{E}^{5}$ as follows

$$
\begin{aligned}
\beta(t)= & \left(\frac{\left(312-25 t^{2}\right)^{5 / 2}}{1521000}, \frac{125 t^{5}-3900 t^{3}+36504 t}{60840},\right. \\
& -\frac{5 t^{2}\left(125 t^{4}-3900 t^{2}+36504\right)}{48672 \sqrt{78}}, \frac{t\left(312-25 t^{2}\right)^{5 / 2}}{243360 \sqrt{78}}, \\
& \left.\frac{1}{4} \sqrt{\frac{23}{78}} t^{2}\right) .
\end{aligned}
$$

With necessary computations we have,

$$
\begin{aligned}
V_{1}^{\beta}(t)=( & -\frac{t\left(312-25 t^{2}\right)^{3 / 2}}{12168}, \frac{625 t^{4}-11700 t^{2}+36504}{60840}, \\
& -\frac{5 t\left(125 t^{4}-2600 t^{2}+12168\right)}{8112 \sqrt{78}}, \frac{\left(52-25 t^{2}\right)\left(312-25 t^{2}\right)^{3 / 2}}{40560 \sqrt{78}}, \\
& \left.\frac{1}{2} \sqrt{\frac{23}{78}} t\right)
\end{aligned}
$$




$$
\begin{aligned}
V_{2}^{\beta}(t)= & \left(\frac{\sqrt{312-25 t^{2}}\left(25 t^{2}-78\right)}{3042}, \frac{5 t\left(25 t^{2}-234\right)}{3042},\right. \\
& -\frac{5\left(625 t^{4}-7800 t^{2}+12168\right)}{8112 \sqrt{78}}, \frac{25 t \sqrt{4-\frac{25 t^{2}}{78}}\left(25 t^{2}-156\right)}{8112}, \\
& \left.\sqrt{\frac{23}{312}}\right)
\end{aligned}
$$

Therefore, the second Frenet vector of the Salkowski curve $\beta$ makes the constant angle $\theta=\arccos \left(\sqrt{\frac{23}{312}}\right)$ with $e_{5}$. The curvature functions of the curve $\beta$ are

$$
\begin{gathered}
k_{1}^{\beta}(t)=1, \\
k_{2}^{\beta}(t)=\sqrt{\frac{4350}{4056-325 t^{2}}-1} \\
k_{3}^{\beta}(t)=\frac{175 \sqrt{6} \sqrt{1625 t^{2}+3732}}{\sqrt{4056-325 t^{2}}\left(325 t^{2}+294\right)} \\
k_{4}^{\beta}(t)=\frac{125 \sqrt{138} t \sqrt{325 t^{2}+294}}{\sqrt{25 t^{2}-312}\left(1625 t^{2}+3732\right)} .
\end{gathered}
$$

Now, we give another method to construct slant helices from an arclength parameterized curve on the unit hypersphere. By means of this method, we can construct rectifying slant helices in $\mathbb{E}^{n}$.

Theorem 3.2. Let $\gamma(u): I \rightarrow E^{n}$ be a unit speed spherical curve on $S^{n-1}$ with

$$
\left\|\frac{d^{2} \gamma(u)}{d u^{2}}\right\|=a
$$

such that

$$
\gamma(u)=\left(\gamma_{1}(u), \gamma_{2}(u), \ldots, \gamma_{n-1}(u), c\right)
$$

where $u(t): I \rightarrow R$ is a differentiable function, $a>1$ and $c \in R /\{0\}$. Then, the curve $\beta$ defined by

$$
\beta(t)=\sec (u(t)) \gamma(u(t))
$$

is a slant helix in $\mathbb{E}^{n}$.

Proof. From the definition of $\gamma$, we have

$$
\|\gamma(u)\|=1, \quad\left\|\frac{d \gamma(u)}{d u}\right\|=1, \quad\left\|\frac{d^{2} \gamma(u)}{d u^{2}}\right\|=a .
$$

Then, we have

$$
\begin{gathered}
\|\beta\|=\sec u \\
\beta^{\prime}=\sec u \quad u^{\prime}\left(\tan u \gamma(u)+\frac{d \gamma(u)}{d u}\right), \\
\left\|\beta^{\prime}\right\|=\sec u^{2} \quad\left|u^{\prime}\right| .
\end{gathered}
$$


With straigtforward calculations, we have

$$
\begin{aligned}
V_{1}^{\beta}= & \sin u \gamma(u)+\cos (u) \frac{d \gamma(u)}{d u} \\
= & \left(\sin u \gamma_{1}(u)+\cos u \frac{d \gamma_{1}(u)}{d u}, \ldots,\right. \\
& \left.\sin u \gamma_{n-1}(u)+\cos u \frac{d \gamma_{n-1}(u)}{d u}, c \sin u\right)
\end{aligned}
$$

and

$$
\begin{aligned}
V_{2}^{\beta} & =\frac{1}{\sqrt{a-1}}\left(\gamma(u)+\frac{d^{2} \gamma(u)}{d u^{2}}\right) \\
& =\frac{1}{\sqrt{a-1}}\left(\gamma_{1}(u)+\frac{d^{2} \gamma_{1}(u)}{d u^{2}}, \ldots, \gamma_{n-1}(u)+\frac{d^{2} \gamma_{n-1}(u)}{d u^{2}}, c\right) .
\end{aligned}
$$

Therefore, $\beta$ is a slant helix whose second Frenet vector makes the constant angle $\theta=\arccos \left(\frac{c}{\sqrt{a-1}}\right)$ with $e_{n}$.

Slant helices that have been constructed from the method in Theorem 3.2 are also rectifying curves. In order to show this, we need the following Corollary which is an immediate consequence of the Theorem 4.4 in [5].

Corollary 3.2. Let $\beta: I \rightarrow \mathbb{E}^{n}$ be a curve with nonzero curvatures. Then, $\beta$ is congruent to a rectifying curve if and only if the tangential component of the position vector of the curve satisfies the equation below

$$
\left\langle\beta(t), V_{1}(t)\right\rangle=\int \nu(t) d t .
$$

Therefore, we have the following Lemma.

Lemma 3.3. The curve $\beta$ defined by Theorem 3.2 is a rectifying slant helix.

Proof. By straightforward calculations, we have

$$
\left\langle\beta(t), V_{1}(t)\right\rangle=\tan (u(t))=\int \nu(t) d t .
$$

From the Corollary 3.2, $\beta$ is a rectifying curve. As we see at Theorem 3.2, this curve is also a slant helix. Therefore, the curve $\beta$ is a rectifying slant helix.

We can construct unit speed slant helices by means of Theorem 3.2 and Corollary 3.2 as follows.

Lemma 3.4. Let $\beta$ be a curve defined by Theorem 3.2. $\beta$ is a unit speed rectifying slant helix if and only if $u(t)=$ $\pm \arctan \left(t+a_{1}\right)$ where $a_{1} \in R$.

Proof. From Equation 3.4, we have

$$
\left\|\beta^{\prime}\right\|=\sec u^{2}\left|u^{\prime}\right|
$$

By solving the differential equation

$$
\sec u^{2}\left|u^{\prime}\right|=1,
$$

we easily have

$$
u(t)= \pm \arctan \left(t+a_{1}\right) .
$$


The following Lemma is a result of Theorem 3.2.

Lemma 3.5. Consider the $W$-curve $\gamma(u): I \rightarrow S^{2 n-1}(P, R) \subset S^{2 n} \subset \mathbb{E}^{2 n+1}$ such that

$$
\gamma(u)=\frac{R}{\sqrt{n}}\left(\sum_{j=1}^{n} \cos \left(c_{j} u\right) e_{2 j-1}+\sum_{j=1}^{n} \sin \left(c_{j} u\right) e_{2 j}\right)+\sqrt{1-R^{2}} e_{2 n+1}
$$

where $u: I \rightarrow R$ is a differentiable function, $P=\left(0,0, \ldots, 0, \sqrt{1-R^{2}}\right) \in \mathbb{E}^{2 n+1}, 0<R=\left(\frac{n}{\sum_{j=1}^{n} c_{j}^{2}}\right)^{1 / 2}<1$ and $c_{i}, c_{j} \in R /\{-1,0,1\}, c_{i} \neq c_{j}, 1 \leq i<j \leq n$. Then, the curve $\beta$ defined by

is a rectifying slant helix in $\mathbb{E}^{2 n+1}$.

$$
\beta(t)=\sec (u(t)) \gamma(u(t))
$$

Proof. The proof of this Lemma is similar to the Lemma 3.1. Therefore, we omit the proof.

Similar to the Corollary 3.1, we have

Corollary 3.3. For the curve $\beta$ in Lemma 3.5, we have the following results;

(i) The rectifying slant helix $\beta$ lies on the cone

$$
\begin{aligned}
C^{2 n}= & \left\{\left(x_{1}, x_{2}, \ldots, x_{2 n+1}\right) \in \mathbb{E}^{2 n+1} \mid\right. \\
& \left.\frac{\left(\sum_{i=1}^{n} c_{i}^{2}\right)}{4 n}\left[\sum_{j=1}^{n}\left(\frac{c_{j}^{2}-1}{c_{j}^{2}}\right)^{2}\left(x_{2 j-1}^{2}+x_{2 j}^{2}\right)-\frac{\sum_{i=1}^{n}\left(\frac{c_{j}^{2}-1}{c_{j}^{2}}\right)^{2}}{-n+\sum_{i=1}^{n} c_{i}^{2}} x_{2 n+1}\right]=0\right\} .
\end{aligned}
$$

(ii) The rectifying slant helix $\beta$ is a geodesic of the cone $C^{2 n}$.

(iii) For $n \geq 2$, if $c_{j}=c_{k}, 1 \leq j, k \leq n, j \neq k$. The slant helix $\beta$ is included in the (2n-1)-dimensional hyperplane of $\mathbb{E}^{2 n+1}$, so we can consider it to be a slant helix in $\mathbb{E}^{2 n-1}$. Also, the rectifying slant helix $\beta$ lies on the cone

$$
C^{2 n-2}=\left\{\left(x_{1}, x_{2}, \ldots, x_{2 n+1}\right) \in C^{2 n} \mid x_{2 j-1}=x_{2 k-1}, x_{2 j}=x_{2 k}\right\} .
$$

(iv) For $n \geq 2$ and $j \in\{1,2, \cdots, n\}$. If $\left|c_{j}\right|=1$, then $(2 j-1)$ th term of the curve $\beta$ vanishes and the (2j)th term of the curve $\beta$ becomes a linear function of the parameter $t$. Therefore, it is included in the $2 n$-dimensional hyperplane of $\mathbb{E}^{2 n+1}$ and its first Frenet vector makes a constant angle with $e_{2 j-1}$. So, we can consider it to be a general helix in $\mathbb{E}^{2 n}$.

Example 3.4. Given that

$$
n=3, \quad u(t)=\arctan (t), \quad c_{1}=\sqrt{2}, \quad c_{2}=\sqrt{3}, \quad c_{3}=\sqrt{5}
$$

in Lemma 3.5, then we have the unit speed rectifying slant helix

$$
\begin{aligned}
\beta(t)=\frac{\sqrt{t^{2}+1}}{\sqrt{10}}( & \cos (\sqrt{2} \arctan (t)), \sin (\sqrt{2} \arctan (t)), \\
& \cos (\sqrt{3} \arctan (t)), \sin (\sqrt{3} \arctan (t)), \\
& \cos (\sqrt{5} \arctan (t)), \sin (\sqrt{5} \arctan (t)), \\
\sqrt{7} &
\end{aligned}
$$

in $\mathbb{E}^{7}$, which is a geodesic of the cone 


$$
\frac{5}{12}\left(x_{1}^{2}+x_{2}^{2}\right)+\frac{10}{9}\left(x_{3}^{2}+x_{4}^{2}\right)+\frac{8}{3}\left(x_{5}^{2}+x_{6}^{2}\right)=\frac{151}{252} x_{7}^{2}
$$

with the second Frenet vector

$$
\begin{aligned}
V_{2}^{\beta}(t)=( & -\frac{\cos (\sqrt{2} \arctan (t))}{2 \sqrt{7}},-\frac{\sin (\sqrt{2} \arctan (t))}{2 \sqrt{7}}, \\
& -\frac{\cos (\sqrt{3} \arctan (t))}{\sqrt{7}},-\frac{\sin (\sqrt{3} \arctan (t))}{\sqrt{7}}, \\
& \frac{2 \cos (\sqrt{5} \arctan (t))}{\sqrt{7}},-\frac{2 \sin (\sqrt{5} \arctan (t))}{\sqrt{7}}, \\
& \left.\frac{1}{2}\right)
\end{aligned}
$$

Therefore, the second Frenet vector of the rectifying slant helix $\beta$ makes the constant angle $\theta=\arccos \left(\frac{1}{2}\right)$ with $e_{7}$. The curvature functions of the curve $\beta$ are

$$
\begin{aligned}
& k_{1}^{\beta}(t)=\frac{\sqrt{\frac{14}{5}}}{\left(t^{2}+1\right)^{3 / 2}}, \\
& k_{2}^{\beta}(t)=\frac{\sqrt{235 t^{2}+39}}{\sqrt{70}\left(t^{2}+1\right)^{3 / 2}}, \\
& k_{3}^{\beta}=\frac{\sqrt{\frac{15}{14}} \sqrt{25239 t^{2}+11323}}{\sqrt{t^{2}+1}\left(235 t^{2}+39\right)}, \\
& k_{4}^{\beta}(t)=\frac{\sqrt{546} \sqrt{235 t^{2}+39} \sqrt{6086 t^{2}+335}}{\left(t^{2}+1\right)\left(25239 t^{2}+11323\right)}, \\
& k_{5}^{\beta}(t)=\frac{\sqrt{\frac{10}{13}} \sqrt{595 t^{2}+271} \sqrt{25239 t^{2}+11323}}{6086 t^{4}+6421 t^{2}+335}, \\
& k_{6}^{\beta}(t)=\frac{4 \sqrt{\frac{35}{13}} t \sqrt{6086 t^{2}+335}}{595 t^{4}+866 t^{2}+271} .
\end{aligned}
$$

By means of the methods that introduced in this work, we can find different families of slant helices which lie on other hypersurfaces.

\section{References}

[1] Ahmad TA, Turgut M. Some characterizations of slant helices in the Euclidean space $E^{n}$. Hac J Math Sta 2010; 39: 327-336.

[2] Arslan K, Celik Y, Deszcz C, Ozgur C. Submanifolds all of whose normal sections are W-curves. Far East J Math Sci 1997; 5: 537-544.

[3] Altunkaya B, Aksoyak FK, Kula L, Aytekin C. On rectifying slant helices in Euclidean 3-space. Kon J Math 2016; 4: 17-24.

[4] Altunkaya B, Kula L. General helices that lie on the sphere $S^{2 n}$ in Euclidean space $E^{2 n+1}$. Uni J Math App 2018; 1: 166-170.

[5] Camci C, Ilarslan K, Kula L, Hacisalihoglu HH. Harmonic cuvature and general helices, Chaos Solitons Fractals 2009; 40: 2590-2596.

[6] Cambie S, Goemans W, Van Den Bussche I. Rectifying curves in the $n$-dimensional Euclidean space, Turkish J Math 2016; 40: 210-223.

[7] Chen BY. When does the position vector of a space curve always lie in its rectifying plane?, Amer Math Monthly 2003; 110: 147-152.

[8] Chen BY, Dillen F. Rectifying curves as centrodes and extremal curves. Bull Inst Math Aca Sinica 2005; 33: 77-90.

[9] Chen BY. Differential geometry of rectifying submanifolds. Int Elec J Geo 2016; 9: 1-8.

[10] Chen BY. Rectifying curves and geodesics on a cone in the Euclidean 3-space. Tamkang J Math 2017; 48: 209-214.

[11] Deshmukh S, Chen BY, Alshammari, SH. On rectifying curves in Euclidean 3-space. Turkish J Math 2017; 42: 609-620.

[12] Gluck H. Higher curvatures of curves in Euclidean space, Amer Math Monthly 1966; 73: 699-704.

[13] Ilarslan K, Nesovic E. Some characterizations of rectifying curves in Euclidean space $E^{4}$, Turkish J Math 2008; 32:21-30.

[14] Izumiya S, Takeuchi N. New special curves and developable surfaces. Turkish J Math 2004; 28: 153-163.

[15] Izumiya S, Takeuchi N. Generic properties of helices and Bertrand curves. J Geom 2002; 74: 97-109. 
[16] Kula L, Yaylı Y. On slant helix and its spherical indicatrix. App Math Comp 2005; 169: 600-607.

[17] Kula L, Ekmekci N, Yaylı Y, Ilarslan K. Characterizations of slant helices in Euclidean 3-space. Turkish J Math 2010; 34 : $261-273$.

[18] Lucas P, Ortega-Yagues JA. Rectifying curves in the three-dimensional sphere. J Math Anal Appl 2015; 421: 1855-1868.

[19] Monterde J. Salkowski curves revisited: A family of curves with constant curvature and non-constant torsion. Com Aided Geo Design $2009 ; 26(3): 271-278$.

[20] O’Neill B. Elementary Differential Geometry. London, UK: Academic Press Inc, 2006.

[21] Salkowski, E. Zur transformation von raumkurven. Mathematische Annalen 1909; 66(4); 517-557.

[22] Yayli Y, Ziplar E. On slant helices and general helices in Euclidean n-space. Mathematica Aeterna 2011; 1: 599-610.

[23] Yayli Y, Gok I, Hacisalihoglu HH. Extended rectifying curves as new kind of modified Darboux vectors. TWMS J Pure Appl Math 2018; 9: 18-31.

\section{Affiliations}

BÜLENT AltunKAYA

AdDress: Ahi Evran University, Faculty of Education

Department of Mathematics, Kirsehir, Turkey.

E-MAIL: bulent.altunkaya@ahievran.edu.tr

ORCID ID : orcid.org/0000-0002-3186-5643 